\title{
MicroED: conception, practice and future opportunities
}

\author{
Tamir Gonen \\ HHMI/UCLA, Los Angeles, United States of America; \\ tgonen@g.ucla.edu
}

My laboratory studies the structures of membrane proteins that are important in maintaining homeostasis in the brain. Understanding structure (and hence function) requires scientists to build an atomic resolution map of every atom in the protein of interest, that is, an atomic structural model of the protein of interest captured in various functional states. In 2013 we unveiled the method Microcrystal Electron Diffraction (MicroED) and demonstrated that it is feasible to determine high-resolution protein structures by electron crystallography of three-dimensional crystals in an electron cryo-microscope (CryoEM). The CryoEM is used in diffraction mode for structural analysis of proteins of interest using vanishingly small crystals. The crystals are often a billion times smaller in volume than what is normally used for other structural biology methods like $\mathrm{x}$-ray crystallography. In this seminar I will describe the basics of this method, from concept to data collection, analysis and structure determination, and illustrate how samples that were previously unattainable can now be studied by MicroED. I will conclude by highlighting how this new method is helping us discover and design new drugs; shedding new light on chemical synthesis and small molecule chemistry; and showing us unprecedented level of details with important membrane proteins such as ion channels and G-protein coupled receptors (GPCRs).

Keywords: MicroED; electron diffraction, cryoem 